AN EVEN SIMPLER DIFFERENTIAL

\author{
DEMAND SYSTEM \\ E. Antony Selvanathan \\ University of Jaffna, Jaffna \\ and \\ Murdoch University, Western Australia \\ Discussion Paper 85.08 \\ July 1985
}

ISSN 0811-6067

ISBN $0 \quad 86422721 \quad 3$ 


\title{
AN EVEN SIMPLER DIFFERENTIAL DEMAND SYSTEM
}

\author{
by \\ E. Antony Selvanathan * \\ University of Jaffna, Jaffna \\ and \\ Murdoch University, Western Australia
}

\begin{abstract}
Keller (1984) proposed three simple variants of the : we1.1-known Rotterdam demand model. This paper proposes an even simpler differential demand system which has some attractive properties and is easy to estimate. The new model is estimated with Dutch and British data.
\end{abstract}

* I an indebted to Professor Kenneth W. Clements of the University of Western Australia for his guidance and comments and to Dr. Kenneth J. Harrison of Murdoch University for his help. 
We write $p_{i}, q_{1}$ for the price and quantity of good $1(1=1, \ldots, n)$, $M=\sum_{i=1}^{n} p_{i} q_{i}$ for income and $w_{i}=p_{i} q_{i} / M$ for the $1^{\text {th }}$ budget share. Under general conditions the relative price version of the differential demand equation for good $i$ can be written as (see Theil, 1980),

$$
w_{i} d\left(\log q_{i}\right)=\theta_{i} d(\log Q)+\sum_{j=1}^{n} v_{i j} d\left(\log \frac{p_{j}}{P^{i}}\right),
$$

where $\theta_{i}=\partial\left(p_{i} q_{i}\right) / \partial M$ is the $i^{\text {th }}$ marginal share; $d(\log Q)=\sum_{i=1}^{n} w_{i} d\left(\log q_{i}\right)$ is the Divisia volume index of the change in the consumer's real income; $d\left[\log \left(\mathrm{p}_{j} / \mathrm{P}^{\prime}\right)\right]$ is the change in the deflated price of $j$, where $d\left(\log P^{\prime}\right)=\sum_{i=1}^{n} \theta_{i} d\left(\log P_{i}\right)$ is the Frisch price index; and

$$
v_{i j}=\frac{\lambda}{M} p_{i} u^{i j} p_{j}
$$

is the $(i, j)^{\text {th }}$ price coefficient, where $\lambda$ is the marginal utility of income and $u^{i j}$ is the $(i, j)^{\text {th }}$ element of the tnverse of the Hessian of the utility function $U=\left[\partial^{2} u / \partial q_{i} \partial q_{j}\right]$. These $v_{i j}{ }^{\prime} s$ satisfy

$$
\sum_{j=1}^{n} v_{i j}=\phi \theta_{1} \quad i=1, \ldots, n,
$$

where $\phi=[\partial(\log \lambda) / \partial(\log M)]^{-1}$ is the income flexibility. It is to be noted that the coefficients of (1) need not be constants.

The absolute price version of (1) is

$$
w_{i} d\left(\log q_{1}\right)=\theta_{1} d(\log Q)+\sum_{j=1}^{n} \pi_{i j} d\left(\log p_{j}\right),
$$


where $\pi_{i j}=v_{i j}-\phi \theta_{i} \theta_{j}$ is the $(i, j)^{\text {th }}$ slutsky coefficient. The $n^{2}$ Slutsky coefficients satisfy demand homogeneity

$$
\sum_{j=1}^{n} \pi_{i j}=0 \quad i=1, \ldots, n
$$

and SIutsky symmetry

$$
\pi_{i j}=\pi_{j i} \quad i, j=1, \ldots, n .
$$

Dividing (4) by $w_{1}$, we obtain $\theta_{i} / w_{1}$ as the income elasticity of demand for $i$ and $\pi_{i j} / w_{i}$ as the $(i, j)^{\text {th }}$ price elasticity. We write these as

$$
\eta_{i}=\frac{\theta_{1}}{w_{i}}, \quad \eta_{i j}=\frac{\pi_{i j}}{w_{i}} .
$$

We propose the simple hypothesis that the larger the budget share of commodity $j$, the larger is the possibility of the consumer switching from commodity $j$ to another commodity $i$ in response to an increase in the $j^{\text {th }}$ price; 1.e., the larger is the budget share of $j$, the higher is the probability that 1 and $j$ are substitutes. In other words, the price elasticity $\eta_{1 j}$ is proportional to the budget share $w_{j}$,

$$
\eta_{i j}=\alpha_{i} w_{j} \quad 1 \neq j
$$

where $\alpha_{i}$ is an 1 -subscripted positive constant. In view of (7), (8) implies $\pi_{i j}=\alpha_{1} w_{1} w_{j}$ for $i \neq j$. It follows from the previous equation and (5) that $-\pi_{11}=\sum_{j \neq i} \pi_{1 j}=\sum_{j \neq 1} \alpha_{1} w_{1} w_{j}=\alpha_{1} w_{1}\left(1-w_{1}\right)$; thus

$$
\alpha_{1}=\frac{-\pi_{i 1}}{w_{1}\left(1-w_{1}\right)}=\frac{-\eta_{11}}{\left(1-w_{1}\right)} .
$$

Equation (6) implies that $\alpha_{i} w_{1} w_{j}=\alpha_{j} w_{j} w_{i}$, from which 1 follows that

$$
\alpha_{1}=\sigma,
$$


a positive constant independent of $i$.

AlI the slutsky coefficients can thus be expressed as $\pi_{i j}=k_{i}\left(\delta_{i j}-w_{j}\right)$, where $k=-\sigma$ and $\delta_{i j}$ is the Kronecker delta, or in matrix form as

$$
\pi=k\left(W-W W^{\prime}\right)
$$

where $\pi=\left[\pi_{1 j}\right], w=\operatorname{diag}\left[w_{1}, \ldots, w_{n}\right]$ and $w=\left[w_{i}\right]$. As $k<0$, we see that $\pi_{i j}=-\kappa w_{1} w_{j}>0$ for $i \neq j$, so that all the goods are substitutes in the Hicksian sense. It can be easily shown that $\pi$ is negative semidefinite when $\sigma>0$, which is in agreement with our hypothesis. As $\pi_{i j} / w_{i} w_{j}=\sigma$ for $i \neq j$, we can interpret $\sigma$ as the cross-elasticity of substitution. Our hypothesis leads to the conclusion that this elasticity is independent of $i$ and $j$; this restriction has been previously used in applied work by Leser (1960) for consumer demand systems and by Powel1 and Gruen (1968) for output supply systems.

Using the definition of $\pi_{i j}$ below equation (4), we can write $v_{i j}=\pi_{i j}+\phi \theta_{i} \theta_{j}$, or $\nu=\pi+\phi \theta \theta^{\prime}$ in matrix form, where $v=\left[v_{i j}\right]$ and $\theta=\left[\theta_{i}\right]$. Using (II), we obtain

$$
v=k\left(W-W W^{\prime}\right)+\phi \theta \theta^{\prime} .
$$

The $i^{\text {th }}$ diagonal element of $v$ is $v_{i i}=k w_{i}-k w_{i}^{2}+\phi \theta_{i}^{2}$; or equivalently, $v_{i i}=\phi w_{i}^{2}\left(\tau\left[\left(1 / w_{i}\right)-1\right]+\eta_{i}^{2}\right)$, where $\tau=k / \phi$. As $\phi<0, k<0$ and $\left(1 / w_{i}\right)-1>0$, we have $v_{i i}<0$. For $i \neq j, \nu_{i j}=\phi w_{i} w_{j}\left(\eta_{i} \eta_{j}-\tau\right)$, indicating that $i$ and $j$ are specific substitutes (complements) according to $\eta_{i} \eta_{j}<\tau(>\tau)$, where this definition of substitutability is from Houthakker (1960). From (2) we can also write $\nu=(\lambda / M) \mathrm{P}^{*} \mathrm{U}^{-1} \mathrm{P}$, where $\mathrm{P}^{*}=\operatorname{diag}\left[\mathrm{p}_{1}, \ldots, \mathrm{p}_{\mathrm{n}}\right]$. Substituting this for $\nu$ in $(12)$ and taking the 
Inverse of both sides, we can show that the $(i, j)^{\text {th }}$ element of $P^{*-1} U P^{*-1}$ (the Hessian matrix of the utility function in expenditure terms) is

$$
\frac{\partial^{2} u}{\partial\left(p_{i} q_{i}\right) \partial\left(p_{j} q_{j}\right)}=\alpha+\beta\left(\frac{\delta_{i j}}{w_{i}}-\left(\eta_{i}+\eta_{j}\right)\right),
$$

where $\alpha=\left(\lambda / M^{2}\right) a_{11}<0 ; \beta=\lambda / M K<0 ; a_{11}=\kappa\left(\kappa+\phi \sum_{i} \theta_{i} \eta_{i}\right) / \phi<0$. It can be easily verified that $\partial^{2} u / \partial\left(p_{i} q_{i}\right) \partial\left(p_{j} q_{j}\right)>0$ iff $\tau<\sum_{k} \theta_{k}^{*} \eta_{k}$, where $\theta_{k}^{*}=-\theta_{k}$ for $k \neq i, j$ and $\theta_{k}^{*}=\left(1-\theta_{k}\right)$ for $k=i, j$, so that $\sum_{k} \theta_{k}^{*}=1$. For the derivation of (13), see the Appendix.

For estimation we make the following adjustments to (4). First, we substitute $k w_{i}\left(\delta_{i j}-w_{j}\right)$ for $\pi_{i j}$. Second, we replace $w_{i}$ with its arithmetic average over the years $t-1$ and $t, \bar{w}_{i t}=1 / 2\left(w_{i t}+w_{i, t-1}\right)$. Third, we replace the infinitesimal logarithmic change in a variable $d(\log x)$ with its finite $\log$-change from $t-1$ to $t, D x_{t}=\log x_{t}-\log x_{t-1}$. Fourth, we take $k$ to be a constant coefficient and use Working's (1943) model for the marginal shares so that $\theta_{i}=\beta_{i}+\bar{w}_{i t}$, where $\beta_{i}$ is a constant with $\sum_{i=1}^{n} \beta_{i}=0$. The substitution term of (4) in terms of finite changes is thus

$$
\sum_{j=1}^{n} k \bar{w}_{i t}\left(\delta_{i j}-\bar{w}_{j t}\right) D p_{j t}=k \bar{w}_{i t}\left(D_{p}-D P_{t}\right)=k \bar{w}_{i t} D p_{i t}^{*},
$$

where $D_{t}=\sum_{i=1}^{n} \bar{w}_{i t} D_{i t}$ is the Divisia price index and $D_{P_{i t}}^{*}=D_{i t}-D_{t}$ is the change in the relative price of $i$. The estimating form of the $i^{\text {th }}$ demand equation is thus

$$
\begin{aligned}
& \bar{w}_{i t}\left(D q_{i t}-D Q_{t}\right)=\beta_{i} D Q_{t}+\kappa \bar{w}_{i t} D_{i t}^{*}+E_{i t}, \quad i=1, \ldots, n, \\
& \mathrm{t}=1, \ldots, \mathrm{T} \text {, }
\end{aligned}
$$

where $D Q_{t}=\sum_{i=1}^{n} \bar{w}_{i t} D_{i t}$ is the finite-change Divisia volume index and 
$\varepsilon_{i t}$ is a zero-mean disturbance. It is to be noted that only the own (Divisia) deflated price appears in each equation; and that (14) is Ifnear in the parameters. Both are highly attractive properties of the mode 1.

Summing both sides of (14) over $1=1, \ldots, n$, we get $\sum_{1=1}^{n} \varepsilon_{1 t}=0$, which Indicates that one of the $n$ equations can be deleted [see Barten (1969)]; we delete the $n^{\text {th }}$. We define the (n-1)T-vector $y=\left[\begin{array}{llll}f_{1}^{\prime} & f_{2}^{\prime} & \ldots & f_{n-1}^{\prime}\end{array}\right]^{\prime}$, with $y_{k}$ as the $k^{t h}$ element, where $f_{1}=\left[\bar{w}_{i 1}\left(D_{i 1}-D Q_{1}\right) \ldots \bar{w}_{i T}\left(D q_{1 T}-D Q_{T}\right)\right]^{\prime}$ is a $T$-vector; the $(n-1) T-$ vector $x_{1 i}$ which has $D_{1}, \ldots, D_{T}$ as elements $(T \times i)+1$ to $T(1+i)$ and zeros elsewhere, with $x_{l i k}$ the $k^{\text {th }}$ element of $x_{11} ;$ and the $(n-1) T-$ vector $x_{2}{ }^{\prime}=\left[g_{1}^{\prime} g_{2}^{\prime} \cdots g_{n-1}^{\prime}\right]^{\prime}$, with $k^{\text {th }}$ element $x_{2 k}$, where $g_{i}=\left[\bar{w}_{i 1} D_{p_{i l}}^{*} \ldots \bar{w}_{i T}{ }^{D p_{i T}^{*}}{ }^{*}{ }^{\prime}\right.$ is a T-vector. We can then write (14) as

$$
y_{k}=\sum_{j=1}^{n-1} \beta_{j} x_{1 j k}+k x_{2 k}+E_{k}, \quad k=1, \ldots,(n-1) T .
$$

Thus as Keller (1984) proposed, (15) can be estimated by oLs by regressing $y_{k}$ on the $n$ explanatory variables $x_{11 k}, \ldots, x_{1, n-1, k}$ and $x_{2 k}$. Although this is a simple procedure, the estimates are not efficient as the disturbances are non-spherical. We thus need to develop the GLS estimator.

We write (14) for $i=1, \ldots, n-1$ as

$$
y_{t}=x_{t} \delta+\varepsilon_{t}, \quad t=1, \ldots, T
$$

where $y_{t}=\left[\vec{w}_{i t}\left(D_{i t}-D Q_{t}\right)\right]$ is an $(n-1)$-vector; $x_{t}$ is a matrix of order $(n-1) \times n$ of which the $i^{\text {th }}$ row is made up of only two non-zero elements, $D Q t$ in the $1^{\text {th }}$ column and $\bar{w}_{1 t^{D}}{ }^{*}{ }_{i t}$ in the $n^{\text {th }}$ column; $\delta=\left[\beta_{1} \cdots \beta_{n-1} \kappa\right]^{\prime} ;$ and $\varepsilon_{t}=\left[\varepsilon_{1 t} \cdots \varepsilon_{n-1 t}\right]^{\prime}$. 
A simple and plausible covariance structure is

$$
\operatorname{var} \varepsilon_{t}=\lambda^{2} \Omega_{t}, \Omega_{t}=W_{t}^{*}-w_{t}^{*} w_{t}^{*}, \quad \operatorname{cov}\left(\varepsilon_{t}, \varepsilon_{s}\right)=0 \text { for } t \neq s,
$$

where $\lambda^{2}$ is a constant, $w_{t}^{*}=\operatorname{diag}\left[\bar{w}_{1 t}, \bar{w}_{2 t}, \ldots, \bar{w}_{n-1, t}\right]$ and $\mathrm{w}_{t}^{*}=\left[\vec{w}_{1 \mathrm{t}} \overline{\mathrm{w}}_{2 t} \cdots \overline{\mathrm{w}}_{\mathrm{n}-1, t}\right]^{\prime}$. It is to be noted that under (17), var $\varepsilon_{t}$ is proportional to the $(n-1) \times(n-1)$ submatrix of the Slutsky matrix $\pi$. This result is implied by the theory of rational random behaviour [see Theil $(1975 / 76,1980)]$. Under $(17)$, the GLS estimator of $\delta$

$$
\hat{\delta}=\left\{\sum_{t=1}^{T} x_{t}^{\prime} B_{t}^{-1} x_{t}\right\}^{-1}\left\{\sum_{t=1}^{T} x_{t}^{\prime} \Omega_{t}^{-1} y_{t}\right\}
$$

and its variance is

$$
\operatorname{var} \hat{\delta}=\lambda^{2}\left\{\sum_{t=1}^{T} x_{t}^{\prime Q}{ }^{-1} x_{t}\right\}^{-1}
$$

The parameter $\lambda^{2}$ can be estimated consistently by

$$
\hat{\lambda}^{2}=\frac{1}{(n-1) T} \sum_{t=1}^{T} \hat{E}_{t}^{\prime \Omega}{ }^{-1} \hat{E}_{t}
$$

where $\hat{\varepsilon}_{t}=y_{t}-x_{t} \hat{\delta}$ is the $(n-1)$-vector of GLS residuals.

Consider the special case when each budget share fin $\Omega_{t}$ is replaced by its sample mean, so that this covariance matrix becomes a constant. The scalar forms of $(18)-(19)$ are then

$$
\hat{\kappa}=\left(z_{0} Z_{Q Q}-B\right) / A z_{Q Q}, \quad \hat{\beta}_{i}=\left(z_{y_{1} Q}-\hat{\kappa} Z_{p_{1} Q}\right) / Z_{Q Q}, \quad 1=1, \ldots, n-1,
$$

and

(22) $\operatorname{var} \hat{\kappa}=\hat{\lambda}^{2} / A, \quad \operatorname{var} \hat{\beta}_{i}=\hat{\lambda}^{2}\left(\mathrm{AZ}_{\mathrm{QQ}} \overline{\mathrm{w}}_{i}\left(1-\overline{\mathrm{w}}_{1}\right)+\mathrm{z}_{\mathrm{p}_{i} \mathrm{Q}}^{2}\right) / \mathrm{AZ} \mathrm{QQ}_{\mathrm{Q}}^{2}, \quad i=1, \ldots, \mathrm{n}-1$, 
where $z_{Q Q}=\sum_{t=1}^{T} D Q_{t}^{2}, \quad z_{p_{i} Q}=\sum_{t=1}^{T} \bar{w}_{i t} D_{i t}^{*} D_{t}^{*}, z_{0}=\sum_{t=1}^{T} \sum_{j=1}^{n} \bar{w}_{j t} D_{j t}^{*} y_{j t} / \bar{w}_{j}$, $y_{i t}=\vec{w}_{i t}\left(D_{i t}-D Q_{t}\right), \quad A=: \sum_{i=1}^{n}\left\{\sum_{t=1}^{T}\left(\bar{w}_{i t} D_{p_{i t}}^{*}\right)^{2}-\left(z_{p_{i} Q}^{2} / z_{Q Q}\right)\right\} / \bar{w}_{i}$, $z_{y_{j} Q}=\sum_{t=1}^{T} y_{j t} D Q_{t}, \quad B=\sum_{j=1}^{n} Z_{p_{j} Q Z_{j} Q^{/ w_{j}}}$ and $\vec{w}_{i}$ is the sample mean of $\overline{\mathrm{w}}_{i t}$. For the derivation of (21) and (22), see the $\Lambda$ ppendix.

As an illustrative application, we use the annual Dutch and British data from Theil (1975/76, Tables 5.2 and 5.3 ) to estimate the model by GLS under (17). The Dutch data cover the period 1922 to 1963 with war years excluded ( $T=3 I$ ) and the British 1900 to 1938 with war years excluded $(T=30)$. There are the same $n=4$ goods in both data sets. Following Theil (1975/76), for the U.K. we added a constant term to each equation to allow for trend-like changes in tastes etc. The estimates are (standard errors in parantheses):

\begin{tabular}{|c|c|c|c|}
\hline & & Netherlands & U.K. \\
\hline \multicolumn{4}{|c|}{ Constant } \\
\hline & $\begin{array}{l}\text { Food } \\
\text { Beverages } \\
\text { Durables } \\
\text { Remainder }\end{array}$ & $\begin{array}{l}- \\
- \\
-\end{array}$ & $\begin{array}{r}.028(.069) \\
-.226(.047) \\
-.001(.057) \\
.199(.070)\end{array}$ \\
\hline \multicolumn{4}{|c|}{$\beta_{1}$} \\
\hline & $\begin{array}{l}\text { Food } \\
\text { Beverages } \\
\text { Durables } \\
\text { Remainder }\end{array}$ & 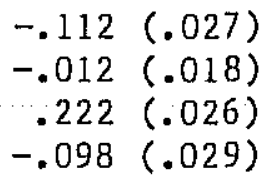 & $\begin{array}{r}-.154(.042) \\
.057(.028) \\
.102(.036) \\
-.004(.043)\end{array}$ \\
\hline$\sigma$ & & $.296(.078)$ & $.560(.062)$ \\
\hline
\end{tabular}

The estimates of $\sigma$ are close to the unweighted averages of the offdiagonal elasticities of substitution at means of .324 and .581, respectively, obtained from the symnetry-constrained estimates given in 
Theil (1975/76, Tables 5.6 and 5.7). When we specify the covariance matrix as a constant, the estimation results remained almost unchanged; for full details, see the Appendix.

\section{REFERENCES}

Barten, A.P. (1969). "Maximum Likelihood Estimation of a Complete System of Demand Equations," European Economic Review 1: 7-73.

Houthakker, H.S. (1960). "Additive Preferences," Econometrica 28: 244-57.

Keller, W.J: (1984). "Some Simple but Flexible Differential Consumer Demand Systems," Economics Letters 16: 77-82.

Leser, C.E.V. (1960). "Demand Functions for Nine Commodity Groups in Australia," Australian Journal of Statistics 2: 102-13.

Powel1, A.A. and F.H.G. Gruen (1968). "The Constant Elasticity of Transformation Production Frontier and Linear Supply System," Internationa1 Economic Review 9: 315-28.

Theil, H. (1975/76). Theory and Measurement of Consumer Demand. Two Vols. Ams terdam: North-Holland.

(1980). The System-Wide Approach to Microeconomics. Chicago: The University of Chicago Press.

Working, H. (1943). "Statistical Laws of Family Expenditure," Journal of the American Statistical Association 38: 43-56. 


\section{APPENDIX}

\section{A Result on Partitioned Inversion}

\section{Proposition:}

Let $\mathrm{D}$ be a non-singular matrix of order $\mathrm{m}$, and consider

$$
X=D+\alpha u u^{\prime}+\beta v v^{\prime},
$$

where $\alpha$ and $\beta$ are scalars and $u$ and $v$ are two m-element vectors. Then

$$
x^{-1}=D^{-1}+D^{-1} u\left(a_{11} D^{-1} u+a_{12} D^{-1} v\right)^{\prime}+D^{-1} v\left(a_{21} D^{-1} u+a_{22} D^{-1} v\right)^{\prime},
$$

where $a_{11}=-\alpha(1+\beta \mu) / r, \quad a_{21}=\alpha \beta \delta / r=a_{12}, \quad a_{22}=-\beta(1+\alpha \gamma) / r$, $r=(1+\alpha \gamma)(1+\beta \mu)-\alpha \beta \delta^{2}, \quad \gamma=\mathrm{u}^{\prime} \mathrm{D}^{-1} \mathrm{u}, \delta=\mathrm{u}^{\prime} \mathrm{D}^{-1} \mathrm{v}$ and $\mu=\mathrm{v}^{\prime} \mathrm{D}^{-1} \mathrm{v}$. provided $r \neq 0$.

Proof: We shall verify that $\mathrm{xx}^{-1}=\mathrm{I}$. We have

$$
\begin{aligned}
x X^{-1}= & \left(D+\alpha u u^{\prime}+\beta v v^{\prime}\right)\left[D^{-1}+D^{-1} u\left(a_{11} D^{-1} u+a_{12} D^{-1} v\right)^{\prime}+D^{-1} v\left(a_{21} D^{-1} u+a_{22} D^{-1} v\right)^{\prime}\right] \\
= & I+u\left(a_{11^{\prime}} D^{-1} u+a_{12} D^{-1} v\right)^{\prime}+v\left(a_{21} D^{-1} u+a_{22} D^{-1} v\right)^{\prime}+\alpha u u^{\prime} D^{-1} \\
& +\alpha u u^{\prime} D^{-1} u\left(a_{11} D^{-1} u+a_{12} D^{-1} v\right)^{\prime}+\alpha u u^{\prime} D^{-1} v\left(a_{21} D^{-1} u+a_{22} D^{-1} v\right)^{\prime}+\beta v v^{\prime} D^{-1} \\
& +\beta v v^{\prime} D^{-1} u\left(a_{11} D^{-1} u+a_{12} D^{-1} v\right)^{\prime}+\beta v v^{\prime} D^{-1} v\left(a_{21} D^{-1} u+a_{22} D^{-1} v\right)^{\prime} \\
= & I+\left(a_{11}+\alpha+\alpha \gamma a_{11}+\alpha \delta a_{21}\right) u u^{\prime} D^{-1}+\left(a_{12}+\alpha \gamma a_{12}+\alpha \delta a_{22}\right) u v^{\prime} D^{-1} \\
& +\left(a_{21}+\beta \delta a_{11}+\beta \mu a_{21}\right) v u^{\prime} D^{-1}+\left(a_{22}+\beta+\beta \delta a_{12}+\beta \mu a_{22}\right) v v^{\prime} D^{-1} \\
= & I+k_{1} u u^{\prime} D^{-1}+k_{2} u v^{\prime} D^{-1}+k_{3} v u^{\prime} D^{-1}+k_{4} v v^{\prime} D^{-1},
\end{aligned}
$$


where

$$
\begin{aligned}
& k_{1}=a_{11}+\alpha+\alpha \gamma a_{11}+\alpha \delta a_{21}, \\
& k_{2}=a_{12}+\alpha \gamma a_{12}+\alpha \delta a_{22}, \\
& k_{3}=a_{21}+\beta \delta a_{11}+\beta \mu a_{21}
\end{aligned}
$$

and

$$
k_{4}=a_{22}+\beta+\beta \delta a_{12}+\beta \mu a_{22} \text {. }
$$

Now we will verify that $k_{1}=k_{2}=k_{3}=k_{4}=0$.

\section{Consider}

$$
\begin{aligned}
k_{1} & =\alpha+a_{11}(1+\alpha \gamma)+\alpha \delta a_{21} \\
& =\alpha-\alpha(1+\beta \mu)(1+\alpha \gamma) / r+\alpha \delta \alpha \beta \delta / r \\
& =\alpha-\alpha\left[(1+\alpha \gamma)(1+\beta \mu)-\alpha \beta \delta^{2}\right] / r \\
& =\alpha-\alpha \\
& =0, \\
k_{2} & =a_{12}+\alpha \gamma a_{12}+\alpha \delta a_{22} \\
& =\alpha \beta \delta(1+\alpha \gamma) / r-\alpha \delta \beta(1+\alpha \gamma) / r \\
& =0, \\
& =0, \\
k_{3} & a_{21}(1+\beta \mu)+\beta \delta a_{11} \\
& \alpha \beta \delta(1+\beta \mu) / r-\beta \delta \alpha(1+\beta \mu) / r \\
& 0,1+\alpha
\end{aligned}
$$




$$
\begin{aligned}
k_{4} & =\beta+(1+\beta \mu) a_{22}+\beta \delta a_{12} \\
& =\beta-\beta(1+\beta \mu)(1+\alpha \gamma) / r+\beta \delta \alpha \beta \delta / r \\
& =\beta-\beta\left[(1+\alpha \gamma)(1+\beta \mu)-\alpha \beta \delta^{2}\right] / r \\
& =\beta-\beta \\
& =0 .
\end{aligned}
$$

Therefore $\mathrm{xx}^{-1}=\mathrm{I}$. This completes the proof.

\section{Derivation of Equation (13)}

From equation (12), we have

$$
\nu=\kappa W-\kappa W^{\prime}+\phi \theta \theta^{\prime}
$$

where $W$ is a non-singular diagonal matrix of order $n$ and $w, \theta$ are $n-$ vectors. Thus using the above proposition,

$$
\begin{aligned}
v^{-1} & =\frac{1}{k} W^{-1}+\frac{1}{k^{2}} W^{-1} w\left(a_{11} W^{-1} w+a_{12} W^{-1} \theta\right)^{\prime}+\frac{1}{k^{2}} W^{-1} \theta\left(a_{21} W^{-1} w+a_{22} W^{-1} \theta\right)^{\prime} \\
& =\frac{1}{k} W^{-1}+\frac{1}{k^{2}} l\left(a_{11^{2}}+a_{12} \eta\right)^{\prime}+\frac{1}{k^{2}} \eta\left(a_{21} 1^{2}+a_{22} \eta\right)^{\prime}, \\
& =\frac{1}{k} W^{-1}+\frac{1}{k^{2}} a_{1 I^{\prime}} l^{\prime}+\frac{1}{k^{2}} a_{12}\left(\imath \eta^{\prime}+\eta \iota^{\prime}\right)+\frac{1}{k^{2}} a_{22} \eta \eta^{\prime},
\end{aligned}
$$

where $\imath^{\prime}=\left[\begin{array}{llll}1 & 1 & \ldots & 1\end{array}\right]^{\prime}, \eta=\left[\eta_{1} \eta_{2} \ldots \eta_{n}\right]^{\prime}, a_{11}=k\left(1+\frac{1}{k} \phi \theta^{\prime} W^{-1} \theta\right) / r$ $=\left(k+\phi \sum_{i=1}^{n} \theta_{i} \eta_{i}\right) / r, a_{12}=-\phi w^{\prime} W^{-1} \theta / r=-\phi / r=a_{21}, a_{22}=-\phi\left(1-w^{\prime} W^{-1} w\right) / r$ $=0$ and $r=\left(1-W^{\prime} W^{-1} w\right)\left(I+\phi \theta^{\prime} W^{-1} \theta\right)+\kappa \phi\left(W^{\prime} W^{-1} \theta / k\right)^{2}=\phi / \kappa \neq 0$. Thus we have

$$
v^{-1}=\frac{1}{k} W^{-1}+\frac{1}{k^{2}} a_{11}{ }^{\prime} \imath^{\prime}-\frac{1}{k}\left(\imath \eta^{\prime}+\eta u^{\prime}\right) .
$$


Therefore if we denote $v^{-1}=\left[v^{i j}\right]$, we have

$$
v^{i j}=\frac{1}{k^{2}} a_{11}+\frac{1}{k w_{i}} \delta_{i j}-\frac{1}{k}\left(\eta_{i}+\eta_{j}\right)
$$

From equation (2), we can write this as

$$
\frac{M}{\lambda} \frac{1}{p_{i} p_{j}} \frac{\partial^{2} u}{\partial q_{i} \partial q_{j}}=\frac{1}{k^{2}} a_{11}+\frac{1}{k w_{i}} \delta_{i j}-\frac{1}{k}\left(\eta_{i}+\eta_{j}\right) .
$$

Therefore,

$$
\frac{\partial^{2} u}{\partial\left(p_{i} q_{i}\right) \partial\left(p_{j} q_{j}\right)}=\alpha+\frac{\beta}{w_{i}} \delta_{i j}-\beta\left(\eta_{i}+\eta_{j}\right),
$$

where $\alpha=\left(\lambda / M k^{2}\right) a_{11} ; a_{11}=k\left(k+\phi \sum_{1} \theta_{i} \eta_{i}\right) / \phi ;$ and $\beta=\lambda / M k$. The above is equation (13). Consider the case $1 \neq j$,

$$
\frac{\partial^{2} u}{\partial\left(p_{i} q_{i}\right) \partial\left(p_{j} q_{j}\right)}=\alpha-\beta\left(\eta_{i}+\eta_{j}\right) \text {. }
$$

Therefore

$$
\begin{aligned}
\frac{\partial^{2} u}{\partial\left(p_{i} q_{i}\right) \partial\left(p_{j} q_{j}\right)}>0 \quad \text { iff } & \alpha>\beta\left(\eta_{i}+\eta_{j}\right) \\
& \text { iff } \frac{\alpha}{\beta}<\left(\eta_{i}+\eta_{j}\right), \quad \text { as } \beta<0 \\
& \text { iff } k+\phi \sum_{i} \theta_{i} \eta_{i}>\phi\left(\eta_{i}+\eta_{j}\right) \quad \text { as } \phi<0 \\
& \text { iff } k>\phi\left[-\sum_{i} \theta_{i} \eta_{i}+\left(\eta_{i}+\eta_{j}\right)\right] \\
& \text { iff } \tau<<\sum_{i} \theta_{i}^{*} \eta_{i},
\end{aligned}
$$

where $\tau=\kappa / \phi$ and

$$
\theta_{k}^{*}= \begin{cases}-\theta_{k} & \text { if } k \neq i, j \\ \left(1-\theta_{k}\right) & \text { if } k=i, j,\end{cases}
$$


13

so that $\sum_{k} \theta_{k}^{*}=1$.

Derivation of (21)-(22)

From equations (18)-(19) we have

$$
\hat{\delta}=\left[\sum_{t=1}^{T} x_{t}^{\prime} \Omega_{t}^{-1} x_{t}\right\}^{-1}\left\{\sum_{t=1}^{T} x_{t}^{\prime} \Omega_{t}^{-1} y_{t}\right\}, \quad \operatorname{var} \hat{\delta}=\lambda^{2}\left\{\sum_{t=1}^{T} x_{t}^{\prime} \Omega_{t}^{-1} x_{t}\right\}^{-1} .
$$

We replace each budget share in $\Omega_{t}$ with its sample mean,

(AI) $\quad \dot{Q}=\overrightarrow{\mathrm{W}}-\overline{\mathrm{WW}}^{\mathrm{T}}$,

where $\overrightarrow{\mathrm{W}}=\operatorname{diag}\left[\overline{\mathrm{w}}_{1}, \overline{\mathrm{w}}_{2}, \ldots, \overline{\mathrm{w}}_{n-1}\right]$ and $\overrightarrow{\mathrm{w}}=\left[\overline{\mathrm{w}}_{1} \overline{\mathrm{w}}_{2} \ldots \overline{\mathrm{w}}_{\mathrm{n}-1}\right]$. If we take the Inverse of both sides of (A1), we get

$$
\Omega^{-1}=\overline{\mathrm{W}}^{-1}+\frac{1}{\overline{\mathrm{W}}_{\mathrm{n}}} u^{1}
$$

Now we consider

$$
\begin{aligned}
& X_{t}^{\prime R}{ }^{-1} X_{t}=\left(\begin{array}{c}
D Q_{t} I_{n-1} \\
p_{t}^{*}
\end{array}\right) s^{-1}\left[D Q_{t} I_{n-1} p_{t}^{*}\right]
\end{aligned}
$$

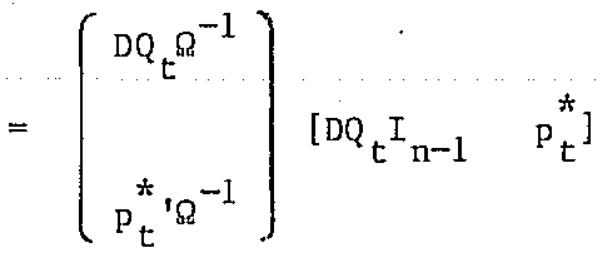

$$
\begin{aligned}
& =\left(\begin{array}{ll}
\mathrm{DQ}_{\mathrm{t}}^{2} \mathrm{\Omega}^{-1} & \mathrm{DQ}_{\mathrm{t}} \mathrm{Q}^{-1} \mathrm{p}_{\mathrm{t}}^{*} \\
\mathrm{DQ} \mathrm{t}_{\mathrm{t}}^{*} \mathrm{~s}^{-1} & \mathrm{p}_{\mathrm{t}}^{*} \mathrm{\Omega}^{-1} \mathrm{p}_{\mathrm{t}}^{*}
\end{array}\right) \text {. }
\end{aligned}
$$


14

where $P_{t}^{*}=\left[\bar{w}_{i t} D_{i t}^{*}\right]$ is a $(n-1)$-vector. Therefore

(A2)

$$
\sum_{t=1}^{T} X_{t}^{\prime} \Omega^{-1} X_{t}=\left[\begin{array}{cc}
z_{Q Q^{\Omega^{-1}}} & \Omega^{-1} z_{p Q} \\
z_{p Q^{\prime}}^{\prime-1} & p^{* *}
\end{array}\right]
$$

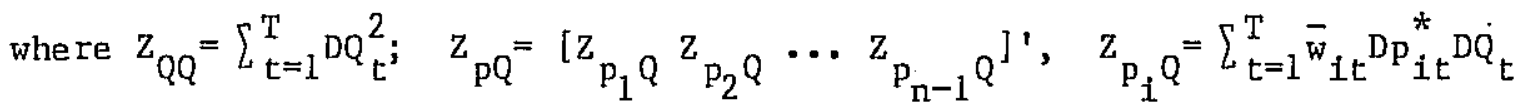
and $\mathrm{p}^{* *}=\sum_{\mathrm{t}=1}^{\mathrm{T}} \mathrm{p}_{\mathrm{t}}^{*} \mathrm{~g}^{-1} \mathrm{p}_{\mathrm{t}}^{*}=\sum_{\mathrm{i}=1}^{\mathrm{n}} \sum_{\mathrm{t}=1}^{\mathrm{T}}\left(\overline{\mathrm{w}}_{i t} \mathrm{Dp}_{i \mathrm{t}}^{*}\right)^{2} / \overline{\mathrm{w}}_{\mathrm{i}}$.

Taking the inverse of both sides of (A2) gives

(AB)

$$
\left\{\sum_{t=1}^{T} x_{t}^{\prime \Omega^{-1}} x_{t}\right\}^{-1}=\left(\begin{array}{cc}
\Omega / z_{Q Q}+z_{p Q} Z_{P Q}^{\prime} / A z_{Q Q}^{2} & -z_{p Q} / A z_{Q Q} \\
-z_{p Q}^{\prime} / A z_{Q Q} & 1 / A
\end{array}\right),
$$

where $A=p^{* *}-\left(z_{p Q}^{\prime}{ }^{-1} z_{p Q}\right) / z_{Q Q}=\sum_{i=1}^{\mathrm{n}}\left\{\sum_{t=1}^{\mathrm{T}}\left(\bar{w}_{i t} D_{p_{i t}^{*}}^{*}\right)^{2}-z_{p_{i}}^{2} / z_{Q Q}\right\} / \vec{w}_{i} \cdot$ Similarly,

$$
X_{t}^{\prime} B^{-1} y_{t}=\left(\begin{array}{c}
D Q_{t}{ }^{-1} y_{t} \\
P_{t}^{*} \Omega^{-1} y_{t}
\end{array}\right)
$$

which gives

(Af)

$$
\sum_{t=1}^{T} x_{t}^{\prime} \Omega^{-1} y_{t}=\left(\begin{array}{l}
\delta^{-1} z_{y Q} \\
\sum_{t=1}^{T} P_{t}^{*} \Omega^{-1} y_{t}
\end{array}\right),
$$

where $z_{y Q}=\left[z_{y_{1} Q} \cdots z_{y_{n-1} Q}\right]^{\prime}, z_{y_{1} Q}=\sum_{t=1}^{T} y_{i t}{ }^{D Q}{ }_{t}$ and $y_{i t}=\bar{w}_{i t}\left(D q_{i t}-D Q t\right)$. 
Thus from (A3) and (A4) we can write

$$
\hat{\delta}=\left[\begin{array}{cc}
\Omega / z_{Q Q}+z_{p Q} Z_{p Q}^{\prime} / A z_{Q Q}^{2} & -z_{p Q} / A Z_{Q Q} \\
-z_{p Q}^{\prime} / A z_{Q Q} & 1 / A
\end{array}\right]\left[\begin{array}{c}
\Omega^{-1} z_{y Q} \\
\sum_{t=1}^{T} P_{t}^{*} \Omega^{-1} y_{t}
\end{array}\right] .
$$

As $\hat{\delta}=[\hat{\beta}, \hat{k}]$ ', we get

(A5)

$$
\begin{aligned}
& \hat{\kappa}=-Z_{p Q}^{\prime} \Omega^{-1} z_{y Q} / A Z_{Q Q}+\sum_{t=1}^{T} P_{t}^{*} \Omega^{-1} y_{t} / A \\
& =-\mathrm{B} / \mathrm{Az} \mathrm{QQ}_{\mathrm{Q}}+\mathrm{Z}_{0} / \mathrm{A} \\
& =\left(z_{0} z_{Q Q}-B\right) / A z_{Q Q} \text {, }
\end{aligned}
$$

where $z_{0}=\sum_{t=1}^{T} p_{t}^{*} \Omega^{-1} y_{t}=\sum_{j=1}^{n}\left\{\sum_{t=1}^{T} \bar{w}_{j t} D_{j t}^{*}{ }_{j t}{ }_{j t} / \vec{w}_{j}\right\}, \quad B=z_{p Q}^{\prime} \Omega^{-1} z_{y Q}$ $=\sum_{j=1}^{n} Z_{p_{j}} Q^{Z} y_{j} Q^{/ \vec{w}_{j}} ;$ and

$$
\begin{aligned}
& \hat{\beta}=\left(\Omega / z_{Q Q}+z_{p Q} Z_{p Q}^{\prime} / A z_{Q Q}^{2}\right)\left(\Omega^{-1} z_{y Q}\right)-z_{p Q}\left(\sum_{t=1}^{T} p_{t}^{*} \Omega^{-1} y_{t}\right\} / A z_{Q Q} \\
& =z_{y Q} / z_{Q Q}+z_{p Q} z_{p Q}^{1} Q^{-1} z_{y Q} / A z_{Q Q}^{2}-z_{p Q}\left[\sum_{t=1}^{T} P_{t}^{*} \Omega^{-1} y_{t}\right\} / A z_{Q Q} \\
& =\mathrm{z}_{\mathrm{yQ}} / \mathrm{z}_{\mathrm{QQ}}+\mathrm{Bz}_{\mathrm{PQ}} / \mathrm{Az}_{\mathrm{QQ}}^{2}-\mathrm{z}_{0} \mathrm{z}_{\mathrm{pQ}} / \mathrm{Az}_{\mathrm{QQ}} \\
& =\left\{z_{y Q}-\left(\left(z_{0} z_{Q Q}-B\right) z_{P Q}\right) / A z_{Q Q}\right\} / z_{Q Q} \\
& =\left(z_{y Q}-\hat{K} z_{p Q}\right) / z_{Q Q} \text {. }
\end{aligned}
$$

Thus we have

(A6)

$$
\hat{\beta}_{i}=\left(z_{y_{i} Q}-\hat{k} z_{p_{i} Q}\right) / z_{Q Q} \quad \text { for } i=1, \ldots, n-1 \text {. }
$$

Equations (A5) and (A6) are identical to equation (21) of the text. 
Using (A3) we have

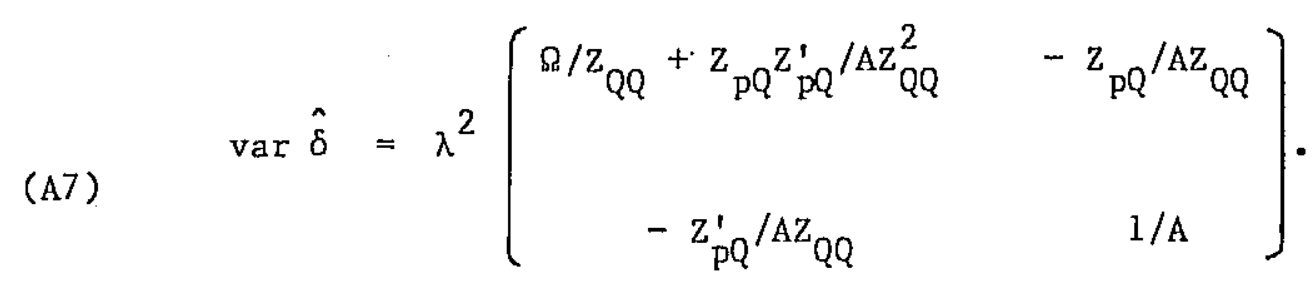

Thus

(A8) $\quad \operatorname{var} \hat{K}=\lambda^{2} / A$

and

$$
\operatorname{var} \hat{\beta}=\lambda^{2}\left(\Omega / Z_{Q Q}+Z_{P Q} Z_{P Q}^{\prime} / A Z_{Q Q}^{2}\right)
$$

or equivalently,

(A9) $\quad \operatorname{var} \hat{\beta}_{i}=\lambda^{2}\left(\mathrm{Az}_{\mathrm{QQ}} \overrightarrow{\mathrm{w}}_{i}\left(1-\overline{\mathrm{w}}_{i}\right)+\mathrm{z}_{\mathrm{p}_{i} \mathrm{Q}}^{2}\right) / \mathrm{Az}_{\mathrm{QQ}}^{2}, \quad i=1, \ldots, \mathrm{n}-1$.

Equations (A8) and (A9) are equivalent to (22).

\section{The Estimation Results}

Below we present the estimates of the model with standard errors given in parantheses and where $\alpha_{i}$ is the constant term in equation $i$ :

\section{Under Specification (17)}

Netherlands (without constants)

U.K. (with constants)

$$
\begin{aligned}
& \beta_{1}=-.112(.027) \\
& \beta_{2}=-.012(.018) \\
& \beta_{3}=.222(.026) \\
& \beta_{4}=-.098(.029) \\
& k=-.296(.078)
\end{aligned}
$$$$
\beta_{1}=-.154(.042)
$$$$
\alpha_{1}=.028(.069)
$$$$
\beta_{2}=.057(.028)
$$$$
\alpha_{2}=-.226(.047)
$$$$
\beta_{3}=.102(.036)
$$$$
\alpha_{3}=-.001(.057)
$$$$
\beta_{4}=-.004(.043)
$$$$
\alpha_{4}=.199(.070)
$$$$
\kappa=-.560(.062)
$$ 
Under Specification of Constant Covariance Matrix

\begin{tabular}{|c|c|c|}
\hline$\beta_{1}=-.111(.027)$ & $\beta_{1}=-.151(.042)$ & $\alpha_{1}=.032(.068)$ \\
\hline$\beta_{2}=-.012(.017)$ & $\beta_{2}=.054(.028)$ & $\alpha_{2}=-.225(.046)$ \\
\hline$\beta_{3}=.222(.026)$ & $\beta_{3}=.104(.036)$ & $\alpha_{3}=-.009(.058)$ \\
\hline$\beta_{4}=-.099(.029)$ & $\beta_{4}=-.007(.043)$ & $\alpha_{4}=.201(.070)$ \\
\hline$\kappa=-.291(.078)$ & $\kappa=-.568(.061)$ & \\
\hline
\end{tabular}

